\title{
Clinicoradiological features of recurrent ischemic stroke: healthcare for poststroke patients
}

\author{
This article was published in the following Dove Press journal: \\ Journal of Multidisciplinary Healthcare \\ 8 July 2010 \\ Number of times this article has been viewed
}

\section{Takehisa Hirayama Yoshikazu Nakamura Yasuhiro Yoshii Ken Ikeda}

Department of Neurology, Toho University Omori Medical Center, Tokyo, Japan
Correspondence: Ken Ikeda Department of Neurology, Toho University Omori Medical Center, 6-I I-I, Omorinishi, Otaku, Tokyo, I43-854I, Japan Tel +8I 337624 I5I

Fax +81337682566

Email keni@med.toho-u.ac.jp
Objective: Little is known about recurrent risk profile of brain infarct (BI) in Japan. The study aimed to clarify clinicoradiological features of recurrent BI patients.

Methods: 374 consecutive BI patients (231 men and 143 women) were admitted to our department between 2007 and 2008. Recurrent BI was defined as a prior history of BI. Cardiovascular disease (CVD) risk profile, stroke mechanism subtypes and Bamford subtypes were compared between the first BI and the recurrent BI group. CVD risk factors, stroke subtypes of the first BI and preventative medication were analyzed in recurrent BI patients.

Results: Recurrent BI existed in 72 patients (40 men and 32 women). Age of the recurrent BI group was significantly older than the first BI group $(P<0.01)$. In comparison to the first BI group, the recurrent BI group had a high frequency of hypertension $(P<0.01)$ and CVD comorbidity $(P<0.05)$. Recurrent rates were increased significantly in cardioembolic patients as compared to the first BI patients $(P<0.05)$. Bamford BI subtypes did not differ between both groups. Mean recurrent interval (SD) was 3.1 (2.3) years. Approximately half the patients experienced recurrent BI less than 2 years after the first BI. Antiplatelet agents were used in 33 patients and warfarin was used in 12 patients as preventative medication. The remaining 27 patients had no prevention therapy by self-cessation. Nine warfarin users were controlled poorly. There were no significant correlations between the first and recurrent Bamford subtypes in recurrent BI patients.

Conclusions: The present study indicated that the recurrent frequency of BI was 19.3\%. Causative profile of recurrent BI suggested elderly age ( $\geq 65$ years), hypertension, prior history of CVD, cardioembolism, 2 years poststroke, insufficient treatment of warfarin and self-cessation of preventative medication. Thus, physicians should pay more attention to these aspects for prophylaxis of recurrent BI in poststroke patients.

Keywords: brain infarct, recurrence, cardiovascular disease risk profile, secondary prevention

\section{Introduction}

Recurrent stroke is an important cerebrovascular event affecting the life of survivors of ischemic stroke. ${ }^{1}$ The second stroke event is an important predictor of functional outcomes as well as mortality after the first stroke. ${ }^{2,3}$ Together with therapeutic development of acute brain infarcts (BI), secondary prevention of $\mathrm{BI}$ is always required in patients with the first BI event. Many prospective studies of recurrent stroke have been reported previously in European and North American populations. ${ }^{1-10}$ Asian prospective studies suggested clinicoradiological hallmarks of recurrent BI. ${ }^{11-14}$ The present retrospective study aimed to elucidate cardiovascular disease (CVD) risk factors and neuroradiological features of recurrent BI in Japanese BI patients. We attempted 
to confirm the importance of preventative healthcare for poststroke patients.

\section{Methods \\ $\mathrm{BI}$ patients}

390 consecutive patients (241 men and 149 women) were diagnosed as BI and admitted to our department between January 2007 and December 2008. Mean age (SD) was 69.6 (12.1) years [67.6 (12.5) in men and 73.0 (10.7) years in women]. We reviewed medical and neuroradiological records. All patients had acute BI within 1 week of clinical onset. BI patients who did not undergo magnetic resonance imaging (MRI) and angiography (MRA) were excluded from the present study. The present study was approved by ethical committee of Toho University Omori Medical Center.

\section{Brain MRI and MRA}

Brain MRI and MRA were performed by a 1.5-tesla superconducting system. Axial T1-weighted imaging, T2-weighted imaging, fluid-attenuated inversion imaging and diffusionweighted imaging (DWI) were obtained. A slice/gap thickness of MRI was $6.0 / 0.5 \mathrm{~mm}$ and the matrix size was $224 \times 256$ for all sequences. The number of acquisitions was 2 . MRA was applied by 3-dimensional time-of-flight technique. A slice thickness was $0.6 \mathrm{~mm}$ and the matrix size was $256 \times 256$ on MRA. Experienced neuroradiologists reviewed brain MRI and MRA. Acute BI lesions were defined as hyperintensity signal areas on DWI and the subsequent new hyperintensity signal areas on T2-weighted imaging.

\section{Diagnosis and preventative medication of recurrent $\mathrm{BI}$}

Among the patients fulfilling the MRI definition mentioned above, recurrent BI was defined as obvious prior history of BI events. The interval between the first and the second BI was assessed. Use of antiplatelet and anticoagulant agents was analyzed in recurrent BI patients. International ratio (INR) of prothrombin time was evaluated in warfarin-treated patients.

\section{CVD risk factors}

CVD risk factors were analyzed on the following 7 items:

- obesity (body mass index $\geq 25.0 \mathrm{~kg} / \mathrm{m}^{2}$ );

- current smoker;

- hypertension (systolic blood pressure $\geq 140 \mathrm{mmHg}$ or diastolic blood pressure $\geq 90 \mathrm{mmHg}$ ) or currently under treatment;

- diabetes mellitus [(DM) fasting blood sugar $\geq 126 \mathrm{mg} / \mathrm{dL}$ or hemoglobin $A_{1 c} \geq 6.5 \%$ ] or currently under treatment;
- dyslipidemia (serum low-density lipoprotein cholesterol $\geq 140 \mathrm{mg} / \mathrm{dL}$ or high-density lipoprotein cholesterol $<40 \mathrm{mg} / \mathrm{dL}$ ) or currently under treatment;

- chronic or paroxysmal atrial fibrillation (AF);

- prior history of CVD.

CVD was defined as ischemic heart diseases, including myocardial infarction and angina pectoris. CVD risk profile was compared between the first and the recurrent BI group.

\section{Neuroradiological assessment}

Stroke mechanism subtype was divided into lacunar infarct (LACI), cardioembolism, atherothrombosis and other. BI topography was classified to four categories according to Bamford subtypes. ${ }^{15}$ LACI; total anterior circulation infarct (TACI); partial anterior circulation infarct (PACI); posterior circulation infarct (POCI). Frequency of stroke mechanism subtype and Bamford subtypes was compared between the first and the recurrent BI group. In recurrent BI patients, stroke mechanism subtype and Bamford subtype of the first BI were analyzed by a medical history and MRI findings.

\section{Statistical analysis}

Statistical analyses of CVD risk factors and neuroradiological data used unpaired Student's T-test, $\chi^{2}$ test and one-way analysis of variance. All significance levels were set at 0.05 .

\section{Results}

\section{Frequency of recurrent $\mathrm{BI}$}

Among 390 patients, 16 patients (10 men and 6 women) excluded, as brain MRI and MRA were not undergone. Recurrent BI existed in 72 patients ( 40 men and 32 women). Four patients ( 2 men and 2 women) had 3 BI events. Frequency of recurrent BI was $19.3 \%, 17.3 \%$ in men and $22.4 \%$ in women. There were no significant differences of sex between the first and recurrent BI patients (Table 1).

\section{CVD risk profile of recurrent $\mathrm{BI}$ patients}

Frequency of CVD risk factors is listed in Table 1. Mean age (SD) of the recurrent BI group was 74.5 (9.8) years [72.0 (9.8) in men and 77.7 (9.0) in women]. The age of the first BI group was 68.4 (12.3) years [64.7 (13.3) in men and 74.7 (6.9) in women]. The age of the recurrent BI group was significantly older than that of the first BI group $(P<0.01)$. The frequency of elderly patients ( $\geq 65$ years) was increased in the recurrent BI group, in comparison with the first $\mathrm{BI}$ group $(P<0.01)$. In comparison to the 
Table I CVD risk profile in the first and recurrent BI group

\begin{tabular}{llll}
\hline & First BI group $(\mathbf{n}=\mathbf{3 0 2})$ & Recurrent BI group $(\mathbf{n}=\mathbf{7 2})$ & All patients $(\mathbf{n}=\mathbf{3 7 4})$ \\
\hline Men/women & $191 / \mathrm{III}$ & $40 / 32$ & $23 \mathrm{I} / \mathrm{I} 43$ \\
Mean age (SD) years & $68.4(12.3)$ & $74.5(9.8)^{* * *}$ & $69.6(I 2.1)$ \\
Hypertension & $186(61.6 \%)$ & $56(77.8 \%)^{* * *}$ & $242(64.7 \%)$ \\
Obesity & $69(22.8 \%)$ & $20(27.8 \%)$ & $89(23.8 \%)$ \\
Dyslipidemia & $71(23.5 \%)$ & $19(26.4 \%)$ & $90(24.1 \%)$ \\
Smoking history & $175(57.9 \%)$ & $32(44.4 \%)$ & $207(55.3 \%)$ \\
Diabetes mellitus & $92(30.5 \%)$ & $22(30.6 \%)$ & $114(30.5 \%)$ \\
Atrial fibrillation & $66(21.9 \%)$ & $23(31.9 \%)$ & $89(23.8 \%)$ \\
Prior history of CVD & $76(25.2 \%)$ & $27(37.5 \%)^{*}$ & $103(27.5 \%)$ \\
\hline
\end{tabular}

Notes: ${ }^{*} P<0.05$; ${ }^{* *} P<0.0$ I between the first and the recurrent $\mathrm{BI}$ group.

Abbreviations: CVD, cardiovascular disease; BI, brain infarct.

first BI group, hypertension rates were significantly higher in the recurrent BI group $(P<0.01)$. Frequency of prior CVD history was significantly increased in the recurrent BI group more than the first BI group $(P<0.05)$. Other CVD risk factors did not differ between the first and the recurrent BI group.

\section{Interval and preventative medicine of recurrent $\mathrm{BI}$}

Mean interval (SD) between the first and the recurrent BI was 3.1 (2.3) years. Approximately half the patients (52.8\%) experienced recurrent BI within 2 years from previous BI. Of 72 recurrent patients, 33 patients received antiplatelet agents: 19 patients aspirin; 11 patients cilostazol; 3 patients clopidogrel. Warfarin was used in 12 patients. The remaining 27 patients had no preventative medication by self-cessation (Table 2). Chronic AF or prior history of CVD existed in 10 antiplatelet users and 12 warfarin users. Mean INR (SD) was 1.5 (0.3) and INR was less than 1.4 in 9 warfarin users. A total of 36 patients disclosed self-cessation of antiplatelet agents or uncontrolled warfarin medication.

\section{Stroke mechanism and Bamford subtypes of the first and recurrent $\mathrm{BI}$}

There were no significant differences in stroke mechanism and Bamford subtypes between the first and the recurrent BI group (Table 3). The first BI subtypes of recurrent patients are expressed in Table 2. The most common subtype of the first BI was cardioembolism on stroke mechanism and LACI on Bamford subtype. Recurrent rates were increased significantly in cardioembolic patients as compared to the first BI patients $(P<0.05)$. As compared to the first BI patients, significant differences of CVD profile were not found in recurrent cardioembolic patients and LACI patients.

\section{Discussion}

Our retrospective study indicated that the frequency of recurrent BI was $19.3 \%, 17.3 \%$ in men and $22.4 \%$ in women. Approximately half of recurrent BI occurred within 2 years post-BI. Recurrent BI profiles revealed elderly patients, hypertension, prior history of CVD, cardioembolism, uncontrolled preventative medication.

Predictors of recurrent stroke have been reported previously in many prospective studies. ${ }^{1-14}$ The risk of a recurrent cerebrovascular event is highest in the first month and year after a stroke and transient ischemic attack (TIA). Approximate $12 \%$ of patients have a recurrent stroke within the first year

Table 2 Interval time, preventative medication and the first stroke subtypes in recurrent $\mathrm{BI}$ patients

\begin{tabular}{ll}
\hline & Recurrent BI ( $\mathbf{n}=\mathbf{7 2})$ \\
\hline Mean interval (SD) & $3.1(2.3)$ years \\
$\leq$ I year & $22(30.6 \%)$ \\
I-2 years & $16(22.2 \%)$ \\
$\geq 2$ years & $34(47.2 \%)$ \\
Preventative medication & \\
Antiplatelet agents & $33(45.8 \%)$ \\
$\quad$ Aspirin & $19(26.4 \%)$ \\
Cilostazol & $11(15.3 \%)$ \\
Clopidogrel & $3(4.2 \%)$ \\
Warfarin & $12(16.7 \%)$ \\
Self-cessation & $27(37.5 \%)$ \\
Stroke mechanism subtypes & \\
of the first BI & \\
LACl & $25(34.7 \%)$ \\
Cardioembolism & $42(58.4 . \%)^{*}$ \\
Atherothrombosis & $1(1.3 \%)$ \\
Others & $4(5.6 \%)$ \\
Bamford subtypes of the first BI & \\
LACl & $25(34.7 \%)$ \\
TACl & $13(18.1 \%)$ \\
PACl & $20(27.8 \%)$ \\
POCI & $14(19.4 \%)$ \\
\hline
\end{tabular}

Note: ${ }^{~} P<0.05$ between the first and the recurrent $\mathrm{BI}$ group.

Abbreviations: $\mathrm{BI}$, Brain infarct; $\mathrm{LACl}$, lacunar infarct; $\mathrm{TACl}$, total anterior circulation infarct; $\mathrm{PACl}$, partial anterior circulation infarct; $\mathrm{POCl}$, posterior circulation infarct. 
Table 3 Stroke mechanism and Bamford subtypes of the first and recurrent $\mathrm{BI}$ group

\begin{tabular}{llll}
\hline SM subtype & $\begin{array}{l}\text { First BI group } \\
(\mathbf{n}=\mathbf{3 0 2})\end{array}$ & $\begin{array}{l}\text { Recurrent BI } \\
\text { group }(\mathbf{n}=\mathbf{7 2})\end{array}$ & $\begin{array}{l}\text { All patients } \\
(\mathbf{n}=374)\end{array}$ \\
\hline $\mathrm{LACl}$ & $107(35.5 \%)$ & $25(34.7 \%)$ & $132(35.3 \%)$ \\
Cardioembolism & $66(21.9 \%)$ & $23(31.9 \%)$ & $89(23.8 \%)$ \\
Atherothrombosis & $49(16.1 \%)$ & $9(12.5 \%)$ & $58(15.5 \%)$ \\
Others & $80(26.5 \%)$ & $15(20.9 \%)$ & $95(25.4 \%)$ \\
BS & & & \\
LACI & $107(35.5 \%)$ & $25(34.7 \%)$ & $132(35.3 \%)$ \\
TACI & $27(8.9 \%)$ & $4(5.6 \%)$ & $31(8.3 \%)$ \\
PACl & $93(30.8 \%)$ & $23(31.9 \%)$ & $116(31.0 \%)$ \\
POCI & $75(24.8 \%)$ & $20(27.8 \%)$ & $95(25.4 \%)$ \\
\hline A
\end{tabular}

Abbreviations: $\mathrm{BI}$, brain infarct; BS, Bamford subtype; $\mathrm{LACl}$, lacunar infarct; TACl, total anterior circulation infarct; $\mathrm{PACl}$, partial anterior circulation infarct; $\mathrm{POCl}$, posterior circulation infarct, SM, stroke mechanism.

and $30 \%$ experience recurrence over 5 years. ${ }^{5,6}$ In Rochester, Minnesota, the major predictor was DM. ${ }^{7,8}$ In the Northern Manhattan Stroke Study, baseline predictors of recurrent stroke were high alcohol consumption, hypertension at discharge and blood glucose concentration at hospital admission. ${ }^{9}$ The Perth Community Stroke Study also found older age, 75-84 years and DM to be major predictors of recurrent stroke at 5 years. ${ }^{6}$ Baseline predictors of stroke recurrence in the Malmö Stroke Registry were DM, TIA and AF. ${ }^{10}$ Several Asian studies also described recurrent rate and CVD profile in BI patients. ${ }^{11-14}$ Recurrence of ischemic stroke was associated with male patients, hyperlipidemia, AF and smoking in Taiwanese casecontrol study. Frequency of LACI was significantly lower in recurrent BI than initial BI. ${ }^{11}$ Among 8,036 BI hospitalized patients, 395 patients (4.9\%) developed a recurrent stroke within 30 days at poststroke. Recurrence most frequently occurred in atherothrombotic patients $(6.6 \%)$, followed by cardioembolic patients $(6.2 \%)$. A previous study confirmed that hypertension and AF contribute to early ischemic stroke recurrence in Japanese BI patients. ${ }^{12}$ A Chinese cohort study revealed first-year recurrence rate of $11.2 \%$ in the registered BI patients. Hypertension, AF and smoking were associated with increased risk of BI recurrence. ${ }^{13}$ Among 1,732 consecutive patients with first-ever acute stroke, including atherothrombotic infarction, LACI, cardioembolic infarction, other infarction, and brain hemorrhage, the overall recurrence rate within the first year was $6.5 \%$. Patients with cardioembolic infarction (9.0\%) and other type of infarction (9.1\%) had more recurrent strokes within the initial year compared with the other subtypes. AF, ischemic heart disease, history of TIA and disability at discharge were important determinants for stroke recurrence and death. ${ }^{14}$ Therefore, clinical profile of recurrent BI identified the similarity between our study and previous Asian studies. ${ }^{11-14}$
Other clinical predictive factors of embolic BI recurrence in cardioembolic stroke patients exhibited alcohol abuse, hypertension, valvular heart disease and AF. ${ }^{16}$ Hypertension and DM were shown as significant factors of recurrent LACI in 733 consecutive patients with LACI. ${ }^{17}$ We also compared CVD risk factors in the first and the recurrent patients with cardioembolic infarct or LACI. There were no statistical differences of CVD profile in our recurrent cardioembolism or LACI patients. Besides CVD risk factors and stroke subtype, 27 recurrent patients stopped preventative therapy by themselves in the present study. Half the recurrent BI patients received no satisfactory prophylactic medication. Thus, physicians should give strict informed consent of preventative medication to BI patients.

\section{Conclusion}

The present study indicated that recurrent rates of BI were not uncommon in Japanese poststroke patients. Clinical hallmarks of recurrent BI patients suggested older age, hypertension, CVD events, cardioembolic infarct, recent 2 years post-BI, uncontrolled warfarin treatment and self-cessation of medication. Thus, we emphasize that physicians should pay more attention to those high-risk aspects of recurrent BI.

\section{Acknowledgment}

We would like to thank Professor Yasuo Iwasaki, Department of Neurology, Toho University Omori Medical Center for the critical suggestion and support in this study.

\section{Disclosure}

The authors report no conflicts of interest in this work.

\section{References}

1. Sciacca RR, Rundek T, Sacco RL, Elkind MS. Recurrent stroke and cardiac risks after first ischemic stroke: the Northern Manhattan Study. Neurology. 2006;66(5):641-646.

2. Ross GW, Petrovitch H, White LR, et al. Characterization of risk factors for vascular dementia: the Honolulu - Asia Aging Study. Neurology. 1999;53(2):337-343

3. Tatemichi TK, Desmond DW, Paik M, et al. Clinical determinants of dementia related to stroke. Ann Neurol. 1993;33(6):568-575.

4. Hankey GJ. Long-term outcome after ischaemic Stroke/transient ischaemic attack. Cerebrovasc Dis. 2003;16 Suppl 1:14-19.

5. Burn J, Dennis M, Bamford J, et al. Long-term risk of recurrent stroke after a first-ever stroke: The Oxfordshire Community Stroke Project. Stroke. 1994;25(2):333-337.

6. Hankey GJ, Jamrozik K, Broadhurst RJ, et al. Long-term risk of first recurrent stroke in the Perth Community Stroke Study. Stroke. 1998;29(12):2491-2500

7. Petty GW, Brown RD Jr, Whisnant JP, et al. Ischemic stroke subtypes: a population-based study of functional outcome, survival and recurrence. Stroke. 2000;31:1062-1068. 
8. Petty GW, Brown RD Jr, Whisnant JP, et al. Survival and recurrence after first cerebral infarction: A population-based study in Rochester, Minnesota, 1975 through 1989. Neurology. 1998;50(1):208-216.

9. Sacco RL, Shi T, Zamanillo MC, Kargman DE. Predictors of mortality and recurrence after hospitalized cerebral infarction in an urban community: The Northern Manhattan Stroke Study. Neurology. 1994;44(4):626-634.

10. Elneihoum AM, Göransson M, Flake P, Janzon L. Three-year survival and recurrence after stroke in Malmö, Sweden: An analysis of Stroke Registry Data. Stroke. 1998;29(10):2114-2117.

11. Lai SL, Weng HH, Lee M, et al. Risk factors and subtype analysis of acute ischemic stroke. Eur Neurol. 2008;60(5):230-236.

12. Toyoda K, Okada Y, Kobayashi S. Early recurrence of ischemic stroke in Japanese patients: the Japan standard stroke registry study. Cerebrovasc Dis. 2007;24(2-3):289-295.
13. Xu G, Liu X, Wu W, Zhang R, Yin Q. Recurrence after ischemic stroke in Chinese patients: impact of uncontrolled modifiable risk factors. Cerebrovasc Dis. 2007;23(2-3):117-120.

14. Yokota C, Minematsu K, Hasegawa Y, Yamaguchi T. Long-term prognosis, by stroke subtypes, after a first-ever stroke: a hospital-based study over a 20-year period. Cerebrovasc Dis. 2004;18(2):111-116.

15. Bamford J, Sandercock P, Dennis M, Burn J, Warlow C. Classification and natural history of clinically identifiable subtypes of cerebral infarction. Lancet. 1991;337:1521-1526.

16. Arboix A, García-Eroles L, Oliveres M, Massons JB, Targa C. Clinical Predictors of Early Embolic Recurrence in Presumed Cardioembolic Stroke. Cerebrovasc Dis. 1998;8(6):345-353.

17. Arboix A, Font A, Garro C, et al. Recurrent lacunar infarction following a previous lacunar stroke: a clinical study of 122 patients. J Neurol Neurosurg Psychiatry. 2007;78(12):1392-1394.
Journal of Multidisciplinary Healthcare

\section{Publish your work in this journal}

The Journal of Multidisciplinary Healthcare is an international, peerreviewed open-access journal that aims to represent and publish research in healthcare areas delivered by practitioners of different disciplines. This includes studies and reviews conducted by multidisciplinary teams as well as research which evaluates the results or conduct of such teams or

\section{Dovepress}

healthcare processes in general. The journal covers a wide range of areas and welcomes submission from practitioners at all levels, from all over the world. The manuscript management system is completely online and includes a very quick and fair peer-review system. Visit http://www.dovepress.com/testimonials.php to read real quotes from published authors.

Submit your manuscript here: http://www.dovepress.com/journal-of-multidisciplinary-healthcare-journal 